\title{
BMJ Open Perceptions of healthcare professionals and managers regarding the effectiveness of GP-led walk-in centres in the UK
}

\author{
Mubashir Arain, ${ }^{1}$ Susan Baxter, ${ }^{2}$ Jon P Nicholl ${ }^{2}$
}

To cite: Arain M, Baxter S, Nicholl JP. Perceptions of healthcare professionals and managers regarding the effectiveness of GP-led walkin centres in the UK. BMJ Open 2015;5:e008286. doi:10.1136/bmjopen-2015008286

- Prepublication history for this paper is available online. To view these files please visit the journal online (http://dx.doi.org/10.1136/ bmjopen-2015-008286).

Received 23 March 2015 Revised 27 May 2015 Accepted 16 June 2015

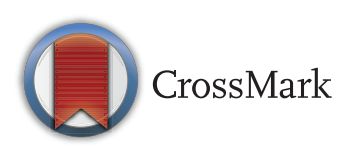

\footnotetext{
${ }^{1}$ Faculty of Nursing, University of Calgary, Calgary, Alberta, Canada ${ }^{2}$ School of Health and Related Research, The University of Sheffield, Sheffield, UK
}

Correspondence to Dr Mubashir Arain; marain@ucalgary.ca

\section{ABSTRACT}

Objectives: This study aimed to identify the perceptions of healthcare professionals regarding the effectiveness and the impact of a new general practitioner-led (GP-led) walk-in centre in the UK. Setting: This qualitative study was conducted in a large city in the North of England. In the past few years, there has been particular concern about an increase in the use of emergency department (ED) services provided by the National Health Service and part of the rationale for introducing the new GP-led walk-in centres has been to stem this increase. The five institutes included in the study were EDs, a minor injuries unit, a primary care trust, a GP-led walk-in centre and GP surgeries.

Participants: Semistructured interviews were conducted with healthcare providers at an adult ED, an ED at a children's hospital, a minor injuries unit, a GPled walk-in centre, GPs from surrounding surgeries and GPs.

Results: 11 healthcare professionals and managers were interviewed. Seven key themes were identified within the data: the clinical model of the GP-led walk-in centre; public awareness of the services; appropriate use of the centre; the impact of the centre on other services; demand for healthcare services; choice and confusion and mixed views (positive and negative) of the walk-in services. There were discrepancies between the managers and healthcare professionals regarding the usefulness of the GP-led walk-in centre in the current urgent care system.

Conclusions: Participants did not notice declines in the demand for EDs after the GP-led walk-in centre. Most of the healthcare professionals believed that the GP-led walk-in centre duplicated existing healthcare services. There is a need to have a better communication system between the GP-led walk-in centres and other healthcare providers to have an integrated system of urgent care delivery.

\section{INTRODUCTION}

The rise in the emergency department (ED) attendances has been of particular concern in the UK over the past few years. ${ }^{1}$ There have been many efforts to strengthen the

\section{Strengths and limitations of this study}

- This is the first study to explore the perceived impact of general practitioner-led (GP-led) walk-in centres in the UK on other urgent care services. The study included a wide range of stakeholders who could identify some potential issues that emerged as a result of the establishment of these centres.

- This study provides valuable information regarding stakeholders' perspectives and organisational implications of establishing GP-led walk-in centres.

- While this is a small sample size, the achievement of data saturation indicates that the sample was adequate to address the research question in this context. The impact of these centres on emergency departments over a period that could be expected with this new service was beyond the scope of this study.

- The qualitative findings from our purposive sample are not intended to be representative, but highlight important insights into barriers and enablers to conduct future large-scale research on the impact of GP-led walk-in centres on other National Health Service.

primary healthcare services and to decrease the unnecessary attendances at EDs. In the past decade, multiple urgent care services have been introduced such as National Health Service (NHS) Direct, urgent dental services, walk-in centres and general practitioner (GP) out-of-hours services to decrease the unnecessary patient load on EDs.

The nurse-led walk-in centres were established in the early 2000s. The aim was to improve patient access to healthcare services for minor illness and injuries by having long opening hours-7:00 to 23:00, 7 days a week. ${ }^{2}$ Some of these centres also employed GPs, but they were mainly led by nurse practitioners. $^{3}$ However, there were concerns about treatment continuity and safety. ${ }^{4}$ 
Moreover, GPs identified the need for better communication between nurse-led walk-in service providers and registered GPs. In contrast to these concerns from GPs, it has been reported that patient satisfaction with the quality of service is greater in nurse-led walk-in centres as a result of easy access and much shorter waiting times compared to GP practices. ${ }^{5}$

The GP-led walk-in centres were introduced in the UK following a report by the Department of Health on the situation of urgent care services. ${ }^{6}$ GP-led walk-in centres aimed to improve patients' access to GPs and to reduce unnecessary patients' visit to EDs. It was also expected that GP-led walk-in centres would address some of the concerns aforementioned about nurse-led walk-in centres. However, it was not clear whether these centres were going to replace the nurse-led centres or established in parallel to the nurse-led centres. The new model was different from the nurse-led model as private healthcare providers operated some of these centres. Moreover, patients could also register with the GPs working at these centres. Around 150 such clinics were planned to open in England. ${ }^{7}$ Although patient satisfaction is reported to be high with the services at these centres ${ }^{8}$ some of the centres have already been closed down after only a few years of operation due to a lack of evidence for reductions in ED attendances. ${ }^{9}$ One report stated that $25 \%$ of GP-led walk-in centres disappeared due to budget cuts in 2012. ${ }^{10} \mathrm{~A}$ report by the King's Fund highlighted an urgent need to evaluate the impact of GP-led walk-in centres. ${ }^{11}$ There were other calls for a greater understanding of the potential role of GP-led walk-in centres in the urgent care system. ${ }^{12}$ Therefore, we conducted a mixed-method evaluation study of a GP-led walk-in centre in the UK. This paper focuses on the qualitative findings. The aim of the qualitative part was to determine the perceptions of healthcare professionals regarding the effectiveness and impact of a GP-led walk-in centre on other local NHS services in a single primary care trust (PCT).

\section{METHODS}

This qualitative exploratory study was designed under the domain of phenomenological approach. ${ }^{13}$ Semistructured interviews were conducted from August 2012 to December 2012. Healthcare providers and healthcare managers were purposively sampled to achieve diversity in professional groups and the range of service locations within a single locality including: an ED at a large city hospital; an ED at a children's hospital; a minor injuries unit; the GP-led walk-in centre; GPs from surrounding surgeries and GPs from an academic unit of primary medical care. Participants were recruited until no new themes emerged (ie, data saturation).

MA conducted hour-long (on average) individual interviews in the participants' offices using a semistructured interview guide developed by the authors, based on a previous study ${ }^{4}$ and also from informal discussions with the local PCT and GP-led walk-in centre manager. A one-hour appointment for the interview was requested, so the duration of interviews was predetermined. The interview guide covered the following topics: introduction to the participants' role in the NHS and any role in relation to the GP-led walk-in centre; perceptions about the services provided at the GP-led walk-in centre; perceived effectiveness and impact of the GP-led walk-in centre on other services; and awareness of the general public about the services provided at the GP-led walk-in centre. All interviews were digitally audiorecorded and then transcribed into word format. Field notes were also taken.

The coding was primarily carried out by MA, with input from SB to discuss emerging codes and data within each theme, prior to discussing data with other members of the research team to establish consensus. Data were coded by hand using thematic analysis to identify recurring themes. Thematic analysis has been increasingly used in health services research. ${ }^{14}$ Each interview was read line by line to identify ideas or concepts within the text. Similar ideas or concepts across the transcripts were brought together and given a descriptive code. Each code was then further examined to develop themes and subthemes within the data set.

Informed written consent was obtained from all participants.

\section{RESULTS}

Eleven participants were interviewed. The participants were broadly from two categories: first, healthcare professionals (consultants, GPs, nurses) and second, managers (GP-led walk-in centre managers, PCT managers). All the participants from the managerial group were either directly or indirectly involved in managing or commissioning the GP-led walk-in centre services. Of the non-managerial group, two healthcare professionals were from a minor injuries unit, two GPs were from surgeries near the walk-in centre, one participant was a GP practice coordinator/manager working within a mile radius of the walk-in centre, and three were doctors from the ED of the local hospital. Table 1 details the characteristics of participants.

On exploration of the perceptions, seven recurring themes were identified that were directly or indirectly related to how local healthcare providers and managers perceived the role of the GP-led walk-in centre in the urgent care system. The themes include uncertainty regarding the clinical model of the GP-led walk-in centre; lack of public awareness about the services provided; uncertainty regarding the impact of the GP-led walk-in centre on other NHS services; increasing demand for healthcare services; concerns regarding appropriate use of the centre; the creation of choice and confusion; and mixed views of the services provided (figure 1 illustrates themes and subthemes within the data).

Some quotes have been subject to minor editing (edits shown in brackets) to clarify the meaning of 


\section{Table 1 Participant characteristics}

\begin{tabular}{ll}
\hline Characteristic & $\mathbf{n}(\%)$ \\
\hline Female & $4(36.4)$ \\
Experience (years) & $3(27.3)$ \\
$0-5$ & $3(27.3)$ \\
$5-10$ & $5(45.5)$ \\
$>10$ & \\
Organisation of work & $1(9.1)$ \\
GP-led walk-in centre & $2(18.2)$ \\
PCT & $2(18.2)$ \\
ED minor injuries unit & $3(27.3)$ \\
ED & $3(27.3)$ \\
GP surgeries near the walk-in centre & $n /$ total \\
Role & $1 / 1$ \\
Operational manager (GP-led walk-in & \\
centre) & $2 / 2$ \\
PCT manager & $2 / 5$ \\
ED nurse practitioner & $1 /$ unknown \\
ED Registrar & $2 / 6$ \\
ED consultant & $2 / 4$ \\
GP & $1 / 1$ \\
GP practice manager & \\
${ }^{*}$ Total shows the denominator, which was the number of potential \\
participants available in each professional group. \\
ED, emergency department; GP, general practitioner; PCT, \\
primary care trust.
\end{tabular}

extracts and to maintain the anonymity of the participants. Furthermore, the source has been referred to only by the group in order to preserve anonymity.

\section{Uncertainty regarding the clinical model}

The participants described differing understandings regarding the existing model of the local GP-led walk-in centre and their preferred model.

\section{Nurse-led versus GP-led}

Some participants perceived that the GP-led walk-in centre was typically nurse-led and only made referrals to a GP when needed. Others believed that a triage nurse decides if a patient is going to see a doctor or a nurse. One participant reported that the payment received by the GP-led walk-in centre was to run a nurse-led service; however, if nurses were unavailable, the service would provide GPs without adding any extra cost to the PCT.

\section{Service provided}

Most of the participants viewed the GP-led walk-in centre model as a GP service with extended hours.

They [the GP-led walk-in centre] usually provide GP services. So all the general GP services really...but walk-in... in a sense that really they are there for people who don't have access to GP whether because of problems with their appointments of by virtue of whether they are within or they are not within reach of GP. (Health Care Professional)
One participant provided an example showing the lack of understanding of GPs about the services provided at the GP-led walk-in centre.

GP practice sent patient here for some blood results on a Saturday; we have people sending for ear syringing, that kind of things, which are not appropriate for a walk-in setting. (Manager)

\section{The model of private providers}

The GP-led walk-in centre in the study area was operated by a private healthcare provider and participants were unhappy about the service being provided by a private company.

I think NHS services taken on by private industry are destined to become then money oriented. (Health Care Professional)

\section{Lack of public awareness about the services provided}

The GP-led walk-in centre had advertised its services in several places such as newspapers, magazines, flyers and local radio; however, most of the participants reported that the publicity was not enough to create awareness in the general public.

In contrast, other participants believed that the advertisement is ineffective for creating awareness in the general public regarding the appropriate use of healthcare services.

I think nobody reads the back of buses or reads pamphlets. (Health Care Professional)

The GPs' role was mentioned to be important in terms of creating awareness for the general public to use the right service in case of an urgent health problem.

I think GP surgeries certainly have a role in educating people about what services are appropriate. (Health Care Professional)

\section{Uncertainty regarding the impact on other NHS services}

The majority of participants were unclear about any impact of the GP-led walk-in centre on reducing patient load in EDs. One GP reported, for example, that the problem of unnecessary attendances at EDs had not been resolved as a result of GP-led walk-in centres.

It is still a problem even if [the GP-led walk-in centre] has reduced it [unnecessary patient load at ED], which I don't know. It has certainly not oversubscribed ED attendance because the problem is still there. (Health Care Professional)

However, participants from the managerial group reported that the centre may have had an impact on unscheduled care services.

[GP-led walk-in centre has] probably a combination of impact...on other GP practices and their out-of-hours GP 
Figure 1 Thematic map of seven major themes and subthemes.

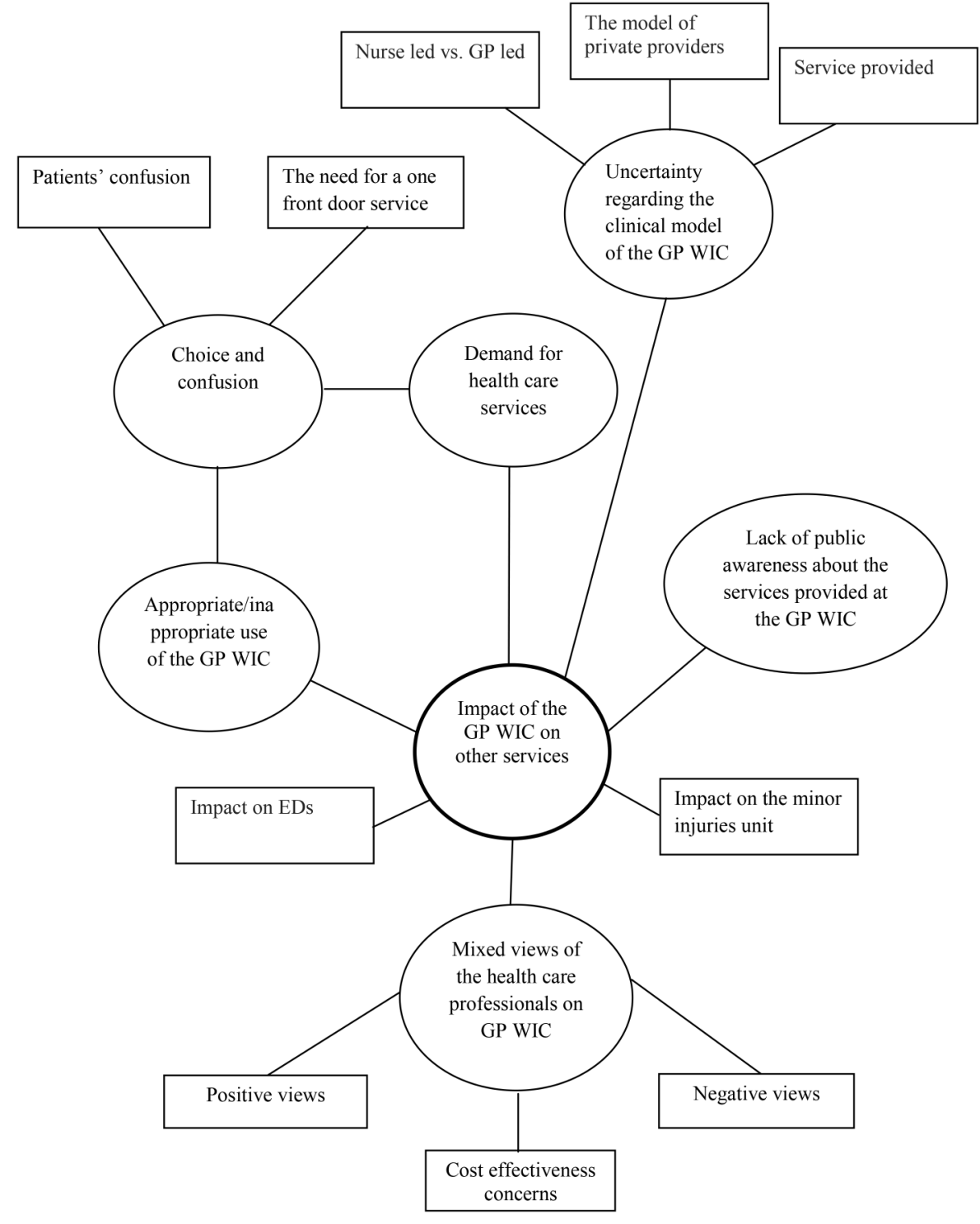

services and the A and Es [accident and emergency departments]. (Manager)

While being uncertain regarding any positive impact, some participants raised concerns regarding a potential increase in the patient load if the GP-led walk-in centre closed down.

The ED buildings are not big enough to cope with the numbers. Remember the GP walk-in centre has around 200 patients per day; that's an awful a lot of extra people for $\mathrm{A}$ and $\mathrm{E}[\mathrm{ED}]$ waiting rooms. (Manager)

I think if you take it [GP-led walk-in centre] out then there will have no choice but to go and see the GP. (Health Care Professional)

\section{Increased demand for healthcare services}

One concern mentioned by participants was the possibility of increased demand for healthcare services resulting from the opening of alternative services such as the GP-led walk-in centre. Participants perceived that the GP-led walk-in centres might have created a demand and would only be fulfilling the demand it created, rather than meeting unmet needs.

$\mathrm{A}$ and $\mathrm{E}$ [ED] has not seen any reduction in their patients. If there is a service, patient[s] will use it. You could have three walk-in centres in the city and all three would be used and you may still not see any dropping in $\mathrm{A}$ and $\mathrm{E}[\mathrm{ED}]$ counts. (Manager)

Another participant mentioned that:

It creates an artificial need and probably does more harm in the long term. (Health Care Professional)

Similarly, it was reported that the GP-led walk-in centre only duplicates services that were already there. 
I think it probably duplicates what a general practice, urgent surgery or emergency surgery would see and what the GP out-of-hours would see. (Health Care Professional)

\section{Concerns regarding appropriate use of the centre}

Participants described the difficulty in labelling a patient visit as an appropriate or inappropriate attendance. It was reported that use of the GP-led walk-in centre may be appropriate if a patient was diverted from the ED, while it may be inappropriate if the patient could have managed the problem without going to any service and only used the centre because it was there.

Patients turning up to the GP walk-in centre wanting ear syringing, which is not something the walk-in bit does. (Manager)

\section{Choice and confusion}

\section{Patient confusion}

Most of the participants perceived that patients get confused about choosing the right healthcare service for their urgent health problems.

I think it's often confusing and difficult for people to decide what service they need; they need some assistance with that. (Health Care Professional)

\section{The need for a 'one front door' service}

Healthcare professionals were in favour of moving all urgent care services to one place and having a 'one front door' service.

I personally would like to see one front door in A and $\mathrm{E}$ [ED] and patients filtered into primary care stream, minor injury stream, or the main A and E department. (Health Care Professional)

Another participant mentioned that it would useful if all services moved to the ED. If all facilities are available at the hospital, it would be easy to manage, and the issue of patients' confusion about choosing the right service could be resolved.

If they [patients] go to one single place, they can be dealt with because of the availability of nurses, doctors, X-rays, blood tests all that kind of thing. I would advocate a single place, one door and one single point of access.

(Health Care Professional)

\section{Mixed (positive and negative) views of the GP-led walk-in centre}

Participants had positive as well as negative views about the GP-led walk-in centre service as well as some views about the cost-effectiveness of the service.

\section{Positive views}

The GP-led walk-in centre was regarded as having improved access to healthcare because of its convenient location.
I think it is easier for patients who are living here [near city centre] to go to GP walk-in centre than to go to GP collaborative or ED, which is at [one] hospital, and is less preferable for patients. (Health Care Professional)

It was also reported that the centre provides a good alternative to the ED if a patient does not have access to a GP. The centre was also perceived to have some positive impact on the local EDs.

Obviously there would be patients who historically would go to an $\mathrm{A}$ and $\mathrm{E}$ [ED] are now going to the GP walk-in centre. I couldn't get an appointment for my daughter and I know she doesn't need to go to children A and E for conjunctivitis so I chose to go to the GP walk-in centre. (Manager)

\section{Negative views}

Healthcare providers had different reasons for having negative views about the GP-led walk-in centre. GPs, for example, reported that they would like to see a service that would complement GP services by providing a walk-in only service, but not a service where patients can register. Healthcare professionals at the minor injuries unit preferred a clearer policy that the centre should not be treating minor injuries cases as it would then only duplicate the services of a minor injuries unit. ED doctors tended to be more in favour of closing down all alternative services and bringing all services at EDs to reduce patients' confusion.

The service [GP-ledwalk-in centre] should not be continuing in the future. I think for several reasons, mainly in the interest of simplifying access to unscheduled care. (Health Care Professional)

GPs were particularly concerned about their list size since the opening of the GP-led walk-in centre.

It [the GP-led walk-in centre] had a negative effect on us. The PCT have established a health care provider within a one hundred and fifty-meter radius of the one that is there for thirty years. Patients can also register there. It definitely has an effect on our registration. Our registration has gone down. (Health Care Professional)

\section{Cost-effectiveness concerns}

Most of the healthcare professionals had concerns regarding the service in terms of value for money.

I think that it's [GP-led walk-in centre] a very expensive service and as far as I understand it, they are paid on a sort of a patient contact or arrived-on-service basis, which means every time a patient walks in they receive a payment for that which is very different to GPs paid in primary care. I don't think it provides good value for money. (Health Care Professional)

\section{DISCUSSION}

The views of the healthcare professionals and managers provide insight into the perceived effectiveness and 
model of care provided by a GP-led walk-in centre in one PCT. There were some discrepancies between the managers and healthcare professionals regarding the usefulness of the GP-led walk-in centre in the current urgent care system. Managers perceived that it was an important service that needed to be continued in the future to prevent any additional burden of patients on EDs. Most of the healthcare professionals, however, were not in support of the idea of alternative urgent services. Previous studies have shown that alternative services in the UK have failed to produce any impact on reducing unnecessary patient load at EDs. ${ }^{15}{ }^{16}$ It has also been reported that alternative healthcare services confuse patients who may not be able to decide which service to choose in case of minor injury/illness. ${ }^{17}$ It is therefore important to address the concerns of all stakeholders before expecting the success of a walk-in service. ${ }^{18}$

The healthcare professionals were clearly in favour of 'one front door' service, which would move all unscheduled care services to an ED. This model has been used elsewhere in the UK where patients with minor problems are redirected from an ED to a co-located nurse-led walk-in centre. ${ }^{19}$ However, one study has shown that co-location of nurse-led walk-in centres with EDs does not reduce patient load at EDs. ${ }^{20}$

Studies have shown that alternative services may not reduce patient load at EDs. ${ }^{21}{ }^{22}$ In contrast, one study conducted on the new model of GP-led walk-in centres has shown a significant reduction in minor illness/injury attendances at an ED after the opening of the centre. ${ }^{23}$

Improvement in patient access to healthcare services has been shown to decrease unnecessary hospital admissions for patients with chronic disease. ${ }^{24}$ Thus, it could be anticipated that the improved access to primary care services would reduce unnecessary patient visits to EDs. GP-led walk-in centres are mostly located in the centre of the city/town, so the geographical access to healthcare services might have improved. Also, functional accessibility has been improved as a result of longer opening hours. Moreover, the opening of the centres on weekends and bank holidays improved accessibility for those who otherwise were less likely to access their GPs.

Unawareness of alternative services is a major reason for not having reduction in the patient load at EDs. One study reported that only a few patients at an ED were aware of an alternative urgent care centre which was co-located with the ED. ${ }^{25}$ Another paper has shown that around half of the patients at an ED were unaware of the existence of the GP out-of-hours or walk-in centre services. ${ }^{21}$ Publicity materials should be available to a wider population through GP surgeries, hospitals and other healthcare services. Publicity material can be improved by clearly indicating the purpose of establishing the GP-led walk-in centre and focusing on the differences between different urgent care services.

Healthcare professionals were unaware of the activity data at the GP-led walk-in centre. There are two possible reasons for this: first, the suboptimal communication between the GP-led walk-in centre and other healthcare providers regarding the available services at the centre, and second, the GP-led walk-in centre does not have an active role in the urgent care services provision. Participants were also concerned about the service being provided by the private sector. The service was free of cost for users and the GP-led walk-in centre charged the NHS for every patient visit. There is a major difference in commissioning a standard GP surgery and GP-led walk-in centre; standard GPs are paid yearly on the number of registered patients while GP-led walk-in centre is paid for per patient visit. The other important concern was that the GP-led walk-in centre was placed in the centre of the city where other GP surgeries were also operating in the surrounding. A few participants reported that the centre was potentially attracting patients who otherwise would attend a GP in the surrounding, creating a competitive environment between a GP-led walk-in centre and other GP surgeries. The services provided by a private healthcare provider created further concern about the competition between standard GP surgeries and the newly established GP-led walk-in centre. Many patients might have registered at the GP-led walk-in centre because of the longer opening hours and easy access. The other GP surgeries were unable to offer $12 \mathrm{~h}$ a day service to compete with the facility that the GP walk-in centre was offering. Thus, measures need to be taken to prevent any decline in the number of registered patients in the surrounding GP surgeries wherever such a centre has been established.

There were some limitations of this study. First, interviews were only conducted in one city, and therefore our findings may not be applicable to other health service locations. Second, only GPs from a surgery near the GP-led walk-in centre were included in the study and other healthcare professionals, such as dental practices and urgent dental care services, were not included. Third, there was small representation from each professional group because of the small study sample. However, our conclusions do not go beyond what the data could support. The findings do not quantitatively determine how many physicians or nurses believed what this paper says, but it does add general perceptions about the centre. Lastly, there was another limitation that most of the analysis and all interviews were done by one person.

\section{CONCLUSION}

Participants did not notice declines in the demand for EDs after the GP-led walk-in centre opened in the locality. Most of the healthcare professionals believed that the GP-led walk-in centre duplicated existing healthcare services. It was also a common belief of healthcare professionals that the general public was unaware of the existing alternative healthcare services and patients often struggle to decide which healthcare service to go to when a GP appointment is unavailable. 
There is a need to have a better communication system between the GP-led walk-in centres and other healthcare providers to have an integrated system of urgent care delivery.

GP-led walk-in centres could potentially attract patients from surrounding GP surgeries. We recommend that future large-scale studies need to examine the impact of GP-led walk-in centres on surrounding GP surgeries as well as on EDs.

Acknowledgements The authors would like to acknowledge the support of the institutes that participated in this research and enabled us to carry out data collection at these sites. They also acknowledge Jill Norris, Faculty of Nursing, University of Calgary, for her support in editing the manuscript.

Contributors JPN and MA planned the overall evaluation project. JPN participated in the design. MA carried out the data collection for the study. SB and MA coded the data and performed thematic analysis with some input from JPN. All the authors read and approved the final manuscript.

Competing interests None declared.

Patient consent Obtained.

Ethics approval Ethical approval for the study was obtained from the Yorkshire and Humber NHS Ethics Committee (reference number: 10/H1304/31).

Provenance and peer review Not commissioned; externally peer reviewed.

Data sharing statement No additional data are available.

Open Access This is an Open Access article distributed in accordance with the Creative Commons Attribution Non Commercial (CC BY-NC 4.0) license, which permits others to distribute, remix, adapt, build upon this work noncommercially, and license their derivative works on different terms, provided the original work is properly cited and the use is non-commercial. See: http:// creativecommons.org/licenses/by-nc/4.0/

\section{REFERENCES}

1. Gillam S. Rising hospital admissions. BMJ 2010;340:275-6.

2. Desborough J, Forrest L, Parker R. Nurse-led primary health care walk-in centres: an integrative literature review. J Adv Nurs 2012;68:248-63.

3. Mabrook AF, Dale B. Can nurse practitioners offer a quality service? An evaluation of a year's work of a nurse led minor injury unit. $J$ Accid Emerg Med 1998;15:266-8.

4. Pope C, Chalder M, Moore L, et al. What do other local providers think of NHS walk-in centres? Results of a postal survey. Public Health 2005;119:39-44.

5. Salisbury C, Manku-Cott T, Moore L, et al. Questionnaire survey of users of NHS walk-in centres: observational study. Br J Gen Prac 2002;52:554-60.

6. Darzi A. High quality care for all: NHS next stage review final report. London: Department of Health, 2008. http://webarchive. nationalarchives.gov.uk/20130107105354/http://www.dh.gov.uk/en/ publicationsandstatistics/publications/publicationspolicyandguidance/ DH_085825

7. Imison C, Naylor C, Maybin J. Under one roof: will polyclinics deliver integrated care? London: King's Fund, 2008.

8. Arain M, Nicholl J, Campbell M. Patients' experience and satisfaction with GP led walk-in centres in the UK; a cross sectional study. BMC Health Serv Res 2013;13:142-51.

9. NHS walk-in centre to close and hours to be cut at another. Yorkshire Post. 27 September 2011. http://www.yorkshirepost.co.uk/ news/nhs-walk-in-centre-to-close-and-hours-to-be-cut-at-another-13811300

10. Wheeler B. Are NHS walk-in centres on the way out? BBC News. 28 June, 2012. http://www.bbc.co.uk/news/uk-politics-18503034

11. Gregory S. General practice in England: an overview. London: King's Fund, 2009. http://www.kingsfund.org.uk/sites/files/kf/Generalpractice-in-England-an-overview-Sarah-Gregory-The-Kings-FundSeptember-2009.pdf

12. Lane J. NHS walk-in centres are dying out. Pharmafield Blog. 3 June, 2013. http://www.pharmafield.co.uk/be/post/2013/06/03/ NHS-walk-in-centres-are-dying-out.aspx

13. Penner JL, McClement SE. Using phenomenology to examine the experiences of family caregivers of patients with advanced head and neck cancer: Reflections of a novice researcher. Int J Qual Methods 2008;7:92-101.

14. Braun V, Clarke V. Using thematic analysis in psychology. Qual Res Psychol 2006;3:77-101.

15. Chalder M, Sharp D, Moore L, et al. Impact of NHS walk-in centres on the workload of other local health care providers: time series analysis. BMJ 2003;326:532-7.

16. Munro J, Sampson F, Nicholl J. The impact of NHS Direct on the demand for out-of-hours primary and emergency care. $\mathrm{Br} J$ Gen Pract 2005;55:790-2.

17. Lattimer V, Burgess A, Knapp F, et al. The impact of changing workforce patterns in emergency and urgent out of hours care on patient experience staff practice and health system performance. Final report for the National Institute for Health Research Service Delivery and Organisation Programme. London: HSMO, 2010.

18. Parker RM, Desborough JL, Forrest LE. Stakeholder perceptions of a nurse led walk-in centre. BMC Health Serv Res 2012;12:382.

19. Lattimer V, Brailsford S, Turnbull J, et al. Reviewing emergency care systems I: insight from system dynamics modelling. Emerg Med $J$ 2004;21:685-91.

20. Salisbury C, Hollinghurst S, Montgomery A, et al. The impact of co-located NHS walk-in centres on emergency departments. Emerg Med J 2007;24:265-569.

21. Penson R, Coleman $\mathrm{P}$, Mason $\mathrm{S}$, et al. Why do patients with minor or moderate conditions that could be managed in other settings attend the emergency department? Emerg Med J 2012;29: 487-91.

22. Coleman $\mathrm{P}$, Irons R, Nicholl J. Will alternative immediate care services reduce demands for non-urgent treatment at accident and emergency? Emerg Med J 2001;18:482-7.

23. Arain M, Campbell M, Nicholl J. Impact of a GP led walk-in Centre on NHS Emergency departments. Emerg Med J 2015;32:295-300.

24. Bindman $\mathrm{AB}$, Grumbach $\mathrm{K}$, Osmond $\mathrm{D}$, et al. Preventable hospitalizations and access to health care. JAMA 1995;274:305-11.

25. Land L, Meredith N. An evaluation of the reasons why patients attend a hospital Emergency Department. Int Emerg Nurs 2013;21:35-41. 